\title{
Enhanced nonradiative relaxation and photoluminescence quenching in random, doped nanocrystalline powders
}

\author{
X. L. Ruan and M. Kaviany ${ }^{\text {a) }}$ \\ Department of Mechanical Engineering, University of Michigan, Ann Arbor, Michigan 48109-2125
}

(Received 8 November 2004; accepted 9 March 2005; published online 13 May 2005)

\begin{abstract}
Nonradiative relaxation and photoluminescence quenching in nanocrystalline powders doped with rare-earth elements are of interest in optical bistability, random laser, and other optoelectronic applications. Here, the luminescence quenching of a one-dimensional random medium made of multilayer nanoparticles $\left(\mathrm{Y}_{2} \mathrm{O}_{3}\right)$ doped with rare-earth elements $\left(\mathrm{Yb}^{3+}\right)$ is analyzed by considering the transport, transition, and interaction of the fundamental energy carriers. The nonradiative decay and luminescence quenching in random media are enhanced compared to single crystals, due to multiple scattering, enhanced absorption, and low thermal conductivity. The coherent wave treatment is used to calculate the photon absorption, allowing for field enhancement and photon localization. The luminescent and thermal emission is considered as incoherent. The size-dependent absorption coefficient and penetration depth are observed. The nonradiative decay is identified as a multiphonon relaxation process, and is found to be enhanced compared to bulk materials. The luminescence quenching and nonlinear thermal emission, occurring with increasing irradiation intensity, are predicted. (c) 2005 American Institute of Physics.
\end{abstract}

[DOI: 10.1063/1.1900937]

\section{INTRODUCTION}

Rare-earth doped solids are noted for their use in highpower lasers, visible emitting phosphors in displays, and other optoelectronic devices. These applications are based on the luminescence emission from intra- $4 f$ shell of the rareearth ions in their $3+$ state. The luminescence emission has been shown to be highly dependent on temperature, ${ }^{1-4}$ due to the temperature dependence of the luminescence quantum yield.

In some rare-earth doped solids irradiated by a laser, the luminescence emission may have two distinct states depending on the increase or decrease in the irradiation intensity (and thus increase or decrease in the temperature). As the irradiation increases, the luminescence emission may be quenched discontinuously (with a jump). This is known as thermo-optical bistability, ${ }^{5}$ and has applications in optical communications, ${ }^{6,7}$ logic and memory systems, ${ }^{8}$ and solidstate lasers. ${ }^{9,10}$ The relationship between luminescence and temperature has been identified as the governing factor of the bistability, and needs to be analyzed carefully.

Recently, luminescence emission in random nanoparticles is of interest, due to their multiple scattering, low thermal conductivity, and radiative trapping properties. Lawandy et $a{ }^{11}{ }^{11}$ observed laserlike luminescence emission from laser dye solutions containing microparticles, suggesting a laser system called random laser. The scattering is thought to create closed paths, or loops, around which the light propagates and becomes amplified in the presence of a population inversion. ${ }^{12}$ In random lasers, thermal effects should be considered carefully, due to the low thermal conductivity of the medium, especially at high temperatures (high power). The

\footnotetext{
a) Author to whom correspondence should be addressed; electronic mail: kaviany@umich.edu
}

effects of temperature dependence of the gain spectrum and the refractive index have been proposed recently. ${ }^{13}$ The luminescence quenching with increasing temperature is also expected to have a considerable impact on the random laser performance.

Most theoretical investigations of the luminescence quenching have been qualitative, and the few available quantitative analyses were performed using a macroscopic, lumped model. Typical treatments are the surface and the bulk treatments. In the former, the absorption, luminescence, and thermal emission are assumed to take place only at the surface. ${ }^{14}$ This is applicable when the extinction coefficient is large and thus the penetration depth is small. In the latter, the absorption is assumed to be lumped and the sample has a uniform temperature. ${ }^{2,410}$ This treatment is usually applicable for solids with a large thermal conductivity. Empirical or fitting parameters are used in both treatments. However, for a sample with small extinction coefficient and small size, the volumetric absorption occurs over a large portion of the penetration depth, and the surface treatment is not suitable. For samples with very low thermal conductivity such as random nanoparticles, the temperature may vary significantly through the sample and cannot be assumed uniform, and the bulk treatment is expected to fail. Here, for the random nanoparticles, a treatment that addresses spatial variations of temperature, absorption, and emission is used.

In this study, the luminescence quenching of random nanoparticles doped with rare-earth elements is analyzed by considering the transport, transition, and interaction of the microscale energy carriers. The coherent wave treatment (Maxwell's equations) is used to calculate the photon absorption, where field enhancement and size-dependent absorption coefficient are observed. Particularly, the nonradiative decay is identified as a multiphonon relaxation process, and is 
Electronic

Excited State

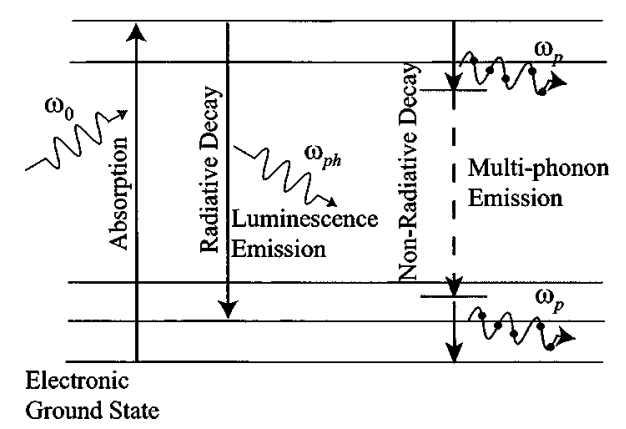

FIG. 1. Interactions among photon, electron, and phonon: photon absorption and emission, electron excitation and decay, and phonon emission.

highly temperature dependent, resulting in luminescence quantum yield rapidly decaying with increase in temperature. The luminescence quenching and nonlinear thermal emission, occurring with increase in irradiation, are then predicted.

\section{THEORETICAL ANALYSIS}

\section{A. Interaction and transport physics}

To understand the luminescence quenching at a microscopic level, the interaction and transport of basic energy carriers must be analyzed. The interaction mechanisms among photon, electron, and phonon in ytterbium-doped yttria are shown in Fig. 1. The process of luminescence in rare-earth systems starts with the absorption of energy on $4 f-4 f$ transitions by electrons, which are normally forbidden, but are rendered by crystal-field mixing. The excited electron may decay by emitting another photon, which is the luminescence emission. This is termed as radiative decay. Due to the Stokes effect, the emitted photon is usually of less energy than the absorbed photon, and some heating takes place. The excited electron may also decay to the ground state by emitting several phonons, and this is termed as nonradiative decay. These radiative and nonradiaive decays compete with each other, and the governing factor is the temperature. The energy spectra of the incident and luminescent photons $\left(E_{\mathrm{ph}, 0}\right.$ and $\left.E_{\mathrm{ph}, l}\right)$, the excited- and ground-state electrons of $\mathrm{Yb}^{3+}$ $\left(E_{e}\right)$, and the optical phonons in yttria $\left(E_{\text {p,optical }}\right)$ are shown in Fig. 2. These will be discussed in detail in Secs. II B-II D. The relative amount of heating is determined by the quantum efficiency $\eta$, which is the ratio of energy radiated to energy absorbed. Generally, $\eta$ is a nonlinear function of temperature. Through these interactions, some photons of the incident frequency disappear, while photons of the luminescent frequency are emitted. Some phonons are also emitted by the nonradiative decay. Electrons are fermions so they cannot be generated or destroyed. However, the population at a particular (excited or ground) state can change by transitions. These interactions provide sources or sinks for the three fundamental energy carriers.

The carrier transports need to be treated in addition to their sources and sinks. For photons, we treat them in a classical regime using Maxwell's equations to allow for the co-

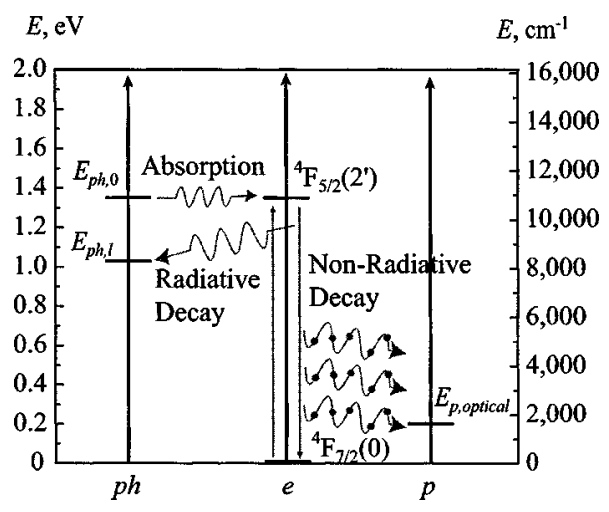

FIG. 2. Energy spectra of the incident (0) and luminescent $(l)$ photons (ph), excited- and ground-state electrons $(e)$, and optical phonons $(p)$, for $\mathrm{Yb}^{3+}: \mathrm{Y}_{2} \mathrm{O}_{3}$ system. The various transitions are also shown.

herent interference effects. Since electrons are bounded to ions and no free electrons are present, we neglect their movement and just consider their transition between the excited and ground states. For phonons, we use the nonradiative decay and the Fourier conduction law, while including the size effect of thermal conductivity in nanomultilayers.

\section{B. Coherent photon absorption}

Absorption occurs when the incident field couples to the dipole moment of the atom. This atom-field interaction is strongest as the incident light is tuned on the resonance of the electronic transition, which is the case in this analysis. Generally the index of extinction is used to describe the absorbing ability of a material. To treat the absorption as a volumetric behavior, the local field amplitude needs to be determined first.

The simplest model of random nanoparticles consists of parallel solid layers with random thickness, as shown in Fig. 3. Regions $l=1$ and $N+1$ are semi-infinite media of air. This multilayer medium has $N / 2$ ( $N$ is an even number here) solid layers and $N / 2-1$ air layers. The coordinates $x_{1}, x_{2}, \ldots, x_{N}$ are chosen such that the thickness of each solid layer is random, but obeys a uniform distribution in the range $\left\langle d_{s}\right\rangle \pm \Delta d_{s}$. This multilayer medium has a finite dimension in the direction of the electromagnetic wave propagation $x$, and an infinite length in the plane normal to $x$. The dielectric solid material has a complex refractive index $m_{s, \omega}\left(=n_{s, \omega}+i \kappa_{s, \omega}\right)$ which depends on the electromagnetic wave frequency. In this section, all the quantities and parameters are at the inci-

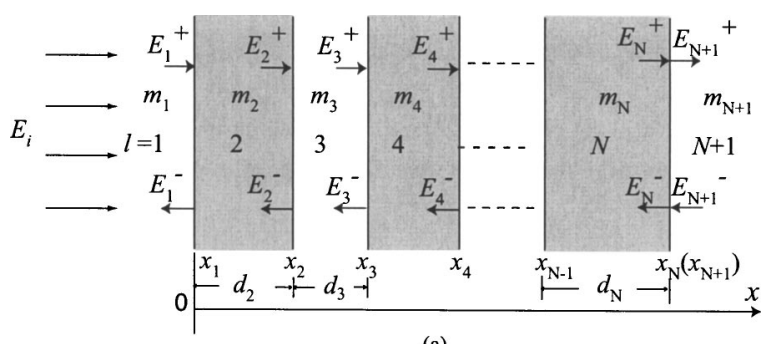

(a)

FIG. 3. Model random nanoparticles, consisting of parallel solid and fluid layers with random thicknesses. The porosity is prescribed. 
dent angular frequency $\omega_{0}$, and thus the subscript $\omega_{0}$ is omitted for convenience. The air has a refractive index $m_{f}\left(=n_{f}\right.$ $=1$ ).

The general theory starts with Helmholtz's equation

$$
\frac{\partial^{2} E(x)}{\partial x^{2}}+k_{0}^{2} m_{l}^{2} E(x)=0,
$$

where $k_{0}$ is the vacuum wave vector, and $m_{l}$ is the local complex index of refraction at the incident frequency. This is the electromagnetic wave equation in a source-free medium, and is equivalent to Maxwell's equations in the multilayer system. For the medium shown in Fig. 3, the solution of Eq. (1) at a particular location in the $l$ th layer is given by

$$
E(x)=E_{l}^{+} e^{i k_{l}\left(x-x_{l}\right)}+E_{l}^{-} e^{-i k_{l}\left(x-x_{l}\right)}, \quad l=1,2, \ldots, N+1,
$$

where $x_{N+1}$ takes the value of $x_{N}$, since there are only $N$ interfaces and $k_{l}=m_{l} \omega_{0} / c_{0}$ is the wave vector, where $c_{0}$ is the speed of light in vacuum. The field in the medium has been divided into two components, the forward (transmitted) component $E_{l}^{+}$and the backward (reflected) component $E_{l}^{-}$. The boundary conditions require that the tangential electric and magnetic fields be continuous across each interface. The relationship between the amplitudes of the $l$ th and $(l+1)$ th interfaces is given in the matrix form ${ }^{15}$

$$
\left(\begin{array}{c}
E_{l}^{+} \\
E_{l}^{-}
\end{array}\right)=D_{l}^{-1} D_{l+1} P_{l+1}\left(\begin{array}{c}
E_{l+1}^{+} \\
E_{l+1}^{-}
\end{array}\right), \quad l=1,2, \ldots, N,
$$

where

$$
D_{l}=\left(\begin{array}{cc}
1 & 1 \\
m_{l} & -m_{l}
\end{array}\right), \quad l=1,2, \ldots, N+1,
$$

and $D_{l}^{-1}$ is the inverse of $D_{l}$, and

$$
P_{l}=\left[\begin{array}{cc}
e^{-i k_{l}\left(x_{l}-x_{l-1}\right)} & 0 \\
0 & e^{i k_{l}\left(x_{l}-x_{l-1}\right)}
\end{array}\right], \quad l=2,3, \ldots, N+1 .
$$

Hence

$$
\left(\begin{array}{c}
E_{j}^{+} \\
E_{j}^{-}
\end{array}\right)=\left[\begin{array}{ll}
M_{11}^{(j)} & M_{12}^{(j)} \\
M_{21}^{(j)} & M_{22}^{(j)}
\end{array}\right]\left(\begin{array}{l}
E_{N+1}^{+} \\
E_{N+1}^{-}
\end{array}\right), \quad j=1,2, \ldots, N,
$$

where

$$
\left[\begin{array}{ll}
M_{11}^{(j)} & M_{12}^{(j)} \\
M_{21}^{(j)} & M_{22}^{(j)}
\end{array}\right]=\prod_{l=j}^{N} D_{l-1} D_{l+1} P_{l+1}, \quad j=1,2, \ldots, N .
$$

For a wave incident from medium 1, we have $E_{N+1}^{-}=0$. Therefore,

$$
\frac{E_{j}^{+}}{E_{1}^{+}}=\frac{M_{11}^{(j)}}{M_{11}^{(1)}}
$$

and

$$
\frac{E_{j}^{-}}{E_{1}^{+}}=\frac{M_{21}^{(j)}}{M_{11}^{(1)}}
$$

The use of Eqs. (8) and (9) in Eq. (2) yields the field everywhere. The magnetic field is then given by ${ }^{16}$

$$
\mathbf{H}(x)=\frac{1}{i \omega_{0} \mu} \nabla \times \mathbf{E}(x),
$$

where $\mu$ is the magnetic permeability.

In order to determine the distribution of the power absorbed inside the layers, the power flux must be determined. The Poynting vector is

$$
I_{0}(x)=|\overline{\mathbf{S}}(x)|=\frac{1}{2}\left|\operatorname{Re}\left[\mathbf{E}(x) \times \mathbf{H}^{*}(x)\right]\right| .
$$

The local energy conversion rate due to the absorption at the resonance frequency is ${ }^{17}$

$$
\dot{s}_{0}(x)=-\frac{\partial I_{0}(x)}{\partial x}=\frac{2 \pi n_{l} \kappa_{l}}{\lambda}\left(\frac{\epsilon_{0}}{\mu_{0}}\right)^{1 / 2}|E(x)|^{2},
$$

where $\epsilon_{0}$ and $\mu_{0}$ are the free space permittivity and permeability.

\section{Rate equation for electrons}

The rate equations describe population dynamics of statistically independent atoms. They are not, however, completely correct when used to describe atoms with any correlations between them, such as in cooperative interactions or other interatomic couplings. In the nanoparticles studied, observation of any cooperative effects is negligible. ${ }^{18}$ In steady state, the excitation rate from the ground state is balanced by the decay rate from the excited state, and the population of the excited state remains constant with respect to time, i.e.,

$$
\frac{d N_{2}}{d t}=\frac{\dot{s}_{0}(x)}{\hbar \omega_{0}}-\gamma(T) N_{2}=0,
$$

where $N_{2}$ is the electronic concentration at the excited state, and $\gamma(T)$ is the temperature-dependent decay rate that will be discussed next.

\section{Nonradiative and radiative decays}

In addition to radiative decays, there can be other mechanisms collectively termed the nonradiative decay. When working with rare-earth elements, the predominant mechanisms are phonon-assisted energy transfer and multiphonon relaxation. Phonon-assisted energy transfer is a nonresonant energy-transfer process in which the mismatch of energy between the level of the sensitizer and the activator is compensated by the simultaneous emission or absorption of one or more phonons. Multiphonon relaxation is decay to a lower level by the emission of two or more phonons. Emission of phonons will lead to internal heating of the system. Miyakawa and Dexter proposed a theory to describe both processes. $^{19}$

The nonradiative decay is through a multiphonon relaxation process, and is governed by the energy-gap law or phonon number law. In measurements on $\mathrm{Yb}^{3+}: \mathrm{Y}_{2} \mathrm{O}_{3}$, the non- 
radiative decay is modeled as a multiphonon process. This is a temperature-dependent rate and the theoretical expression can be given by

$$
\gamma(T)=\gamma(T=0)\left[1-\exp \left(\frac{-\hbar \omega_{p}}{k_{B} T}\right)\right]^{-N},
$$

where $\gamma(T=0)$ is the decay rate at absolute zero temperature, $k_{B}$ is the Boltzmann constant, $\hbar \omega_{p}$ is the energy per phonon, and $N$ is the number of phonons involved in one transition.

The decay rate given in Eq. (14) is the sum of the radiative and nonradiative decay rates. The radiative decay rate is usually assumed to be temperature independent. The nonradiative decay rate is temperature dependent, because it is a multiphonon relaxation process and the phonon occupation number is temperature dependent. At absolute zero temperature, no nonradiative decay exists. The radiative and nonradiative decay rates are thus given by

$$
\gamma_{\mathrm{r}}=\gamma(T=0)
$$

and

$$
\gamma_{\mathrm{nr}}(T)=\gamma(T)-\gamma_{\mathrm{r}}
$$

The energy conversion rate due to the luminescence emission is given by

$$
\dot{s}_{l}=-\gamma_{\mathrm{r}} N_{2} \hbar \omega_{l},
$$

where $\omega_{l}$ is the average luminescent frequency. form

Using Eq. (13) in Eq. (17), $\dot{s}_{l}$ is written in a more explicit

$$
\dot{s}_{l}(x)=-\frac{\omega_{l}}{\omega_{0}} \eta \dot{s}_{0}(x),
$$

where $\eta$ is the luminescent quantum yield and is defined as

$$
\eta=\frac{\gamma_{\mathrm{r}}}{\gamma(T)}
$$

\section{E. Thermal emission}

Thermal emission always exists when a matter is above the absolute zero temperature, and the spectral blackbody emissive power is given by the Planck law. At low temperatures, thermal emission is small, and the detected emission is mainly the rare-earth luminescence emission. However, at high temperatures, the thermal emission becomes dominant. There have been several reports of blackbody emission of nanopowders at high temperatures. Costa et al. ${ }^{20}$ investigated the emission features of silicon nanopowder. The powders were treated as independent blackbody radiators, and the total emission is calculated as the sum of their individual emissions. The emission spectrum was detected at high temperature and was verified to be the blackbody spectrum. Redmond et al. ${ }^{14}$ treated the thermal emission of yttria nanopowders as a surface phenomenon, and the experimentally detected emission spectrum was also demonstrated to be the blackbody spectrum.

It has not been clear in the literature as how this weakly absorbing material $\left(\kappa_{s, \omega_{0}} \sim 10^{-5}\right)$ reveals a blackbody behavior. Here for simplicity we treat the multilayer system as an effective medium with homogeneous properties, and thus the scattering can be neglected. The equation of radiative transfer is ${ }^{21}$

$$
\frac{d I_{t, \lambda}}{d x}=-\sigma_{a, \lambda} I_{t, \lambda}+\sigma_{a, \lambda} I_{b, \lambda},
$$

where $I_{t, \lambda}$ is the spectral intensity of thermal emission, $\sigma_{a, \lambda}$ is the spectral absorption coefficient, and $I_{b, \lambda}$ is the blackbody emission intensity, given by the Planck law. Note that the emission of the excited state is enhanced and is no longer given by the Planck law, but this has already been addressed separately as the luminescence emission.

Equation (20) is integrated from $x=0$ to $x$, starting from an initial intensity $I_{t, \lambda}(0)=0$, and this yields ${ }^{21}$

$$
I_{t, \lambda}(x)=I_{t, \lambda}(x=0) \exp \left(-\sigma_{a, \lambda} x\right)+I_{b, \lambda}\left[1-\exp \left(-\sigma_{a, \lambda} x\right)\right] .
$$

In the multilayer system, the initial thermal emission intensity $I_{t, \lambda}(x=0)$ is zero, and Eq. (21) is simplified to

$$
I_{t, \lambda}(x)=I_{b, \lambda}\left[1-\exp \left(-\sigma_{a, \lambda} x\right)\right] .
$$

To obtain the total thermal emission intensity, an integration over all the wavelengths is to be performed on Eq. (22). Recognizing that the spectral absorption coefficient is wavelength dependent, an average absorption coefficient $\bar{\sigma}_{a}$ which is not dependent on wavelength can be defined, such that the total thermal emission intensity is the same as that using the spectral absorption. Physically, yttria is considered as transparent at all wavelengths except for the transition resonances, however, absorption always exists for all wavelengths due to defects or impurities, etc. Here, for simplicity the average absorption coefficient $\bar{\sigma}_{a}$ is taken as $1 / 10$ of that of the resonance absorption. The total thermal emission is

$$
I_{t}(x)=I_{b}\left[1-\exp \left(-\bar{\sigma}_{a} x\right)\right]
$$

where $I_{b}$ is the total emissive power given by the StefanBoltzmann law. It is clear that if the medium is optically thick $\left(\bar{\sigma}_{a} L\right.$ is large), the collected emission at the surface $(x$ $=L$ ) is nearly the blackbody emission.

Thermal emission could be treated more precisely by not assuming the multilayer system as an effective medium, but including the reflection and transmission at all the interfaces. However, this will be very computing intensive for a large number of layers, and will not change the main results (that the collected emission at the surface is nearly the blackbody emission). ${ }^{21}$ Thus, the effective-medium approximation is used here.

The derivative of the total thermal emission intensity gives the local energy conversion rate due to thermal emission, i.e.,

$$
\dot{s}_{t}(x)=-\frac{d I_{t}}{d x}=-\bar{\sigma}_{a} I_{b} \exp \left(-\bar{\sigma}_{a} x\right) .
$$

Note that the scattering of thermal emission is neglected in the above analysis. Actually, scattering of the thermal emission exists in the nanopowder system, and the localization of thermal emission is possible although the coherence length is small. However, the scattering of thermal emission 
is not important in solving the energy equation and the temperature distribution, since only the absorption of the thermal emission, not the scattering, will appear in the energy equation. On the other hand, it is not practical at this time to tackle the scattering of thermal emission, since the emissivity depends on both the wavelength and the temperature, and the computation would be too heavy to be performed in the multilayer system composed of thousands of layers. As a result, the effective-medium approximation used in this study is an obvious choice.

\section{F. Heat conduction}

Temperature is a critical parameter that governs luminescence quenching. For one-dimensional, steady-state heat conduction, the divergence of the conduction heat flux vector is given by the Fourier conduction law

$$
\nabla \cdot \mathbf{q}_{k}=\nabla \cdot[k(T) \nabla T],
$$

where $k(T)$ is treated as a temperature-dependent thermal conductivity. Here, the sample temperature varies from room temperature $(300 \mathrm{~K})$ to the melting temperature of yttria $(2683 \mathrm{~K})$. The thermal conductivities of yttria and air both change significantly in this range. ${ }^{22}$ Additionally, when the thickness of the gas or solid layer is nearly the same as or smaller than the gas or phonon mean free path, the size effect must be considered. There are simple, approximation expressions describing this effect. One of the models used to predict the size dependence is ${ }^{23}$

$$
k_{f}\left(T, d_{f}\right)=\frac{k_{f}(T)}{1+\frac{4 a_{1}(2-\gamma)}{\gamma\left(c_{p} / c_{v}+1\right)} \mathrm{Kn}_{d_{f}}},
$$

where $a_{1}$ is a semiempirical constant, $0 \leqslant \gamma \leqslant 1$ is the accommodation factor, and $\mathrm{Kn}_{d_{f}}$ is the Knudsen number defined as

$$
\mathrm{Kn}_{d_{f}}=\frac{\lambda_{f}}{d_{f}},
$$

where $d_{f}$ is the fluid layer thickness, and the gas mean free path $\lambda_{f}$ is given by

$$
\lambda_{f}=\frac{1}{2^{1 / 2}} \frac{k_{B} T}{d_{m}^{2} p},
$$

where $d_{m}$ is the gas molecule collision diameter, and $p$ is the pressure.

The solid layer-thickness dependence of the thermal conductivity of the solid layers may also be approximated as ${ }^{23}$

$$
k_{s}\left(T, d_{s}\right)=\frac{k_{s}(T)}{1+\frac{4}{3} \frac{\lambda_{p}}{d_{s}}},
$$

where $k_{s}(T)$ is the bulk solid thermal conductivity, $d_{s}$ is the solid layer thickness, and $\lambda_{p}$ is the phonon mean free path. The predicted thermal conductivities of the gas and solid layers, using Eqs. (26) and (29), are plotted in Fig. 4.

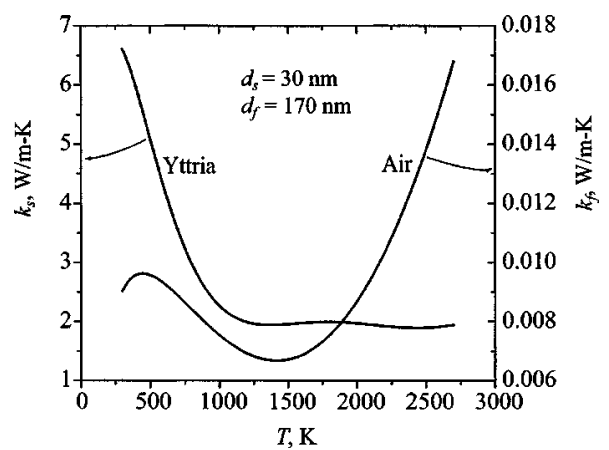

FIG. 4. The solid and gas thermal conductivites as a function of temperature, with the size effects included.

\section{G. Energy conservation}

The last step in completion of the theoretical model is to utilize the energy conservation equation by realizing that the sum of the divergence of the conduction and radiation heat flux vectors should be zero, i.e.,

$$
\nabla \cdot\left(\mathbf{q}_{k}+\mathbf{q}_{\mathrm{r}}\right)=0
$$

where the divergence of the radiative heat flux vector is equal to the volumetric energy conversion due to photon absorption, luminescent, and thermal emission, i.e.,

$$
\nabla \cdot \mathbf{q}_{\mathrm{r}}=\dot{s}_{\mathrm{r}}=\dot{s}_{0}+\dot{s}_{l}+\dot{s}_{t} .
$$

Using Eq. (25), we have

$$
\frac{d}{d x}\left[k(T) \frac{d T}{d x}\right]+\dot{s}_{\mathrm{r}}(x, T)=0,
$$

with the boundary conditions

$$
T=T_{0}, \quad x=x_{N}
$$

and

$$
\frac{d T}{d x}=0, \quad x=0 .
$$

The numerical finite-volume method is used to solve the above equations, and converged solutions are obtained.

\section{RESULTS AND DISCUSSION}

\section{A. Field enhancement and penetration depth}

The above analysis is performed on multilayer systems to predict their luminescent and thermal emission variations with respect to irradiation intensity. The parameters and properties used in the simulation are listed in Table I. The local electric-field component at the irradiation frequency is determined for a normal incident electromagnetic wave of wavelength $\lambda=906 \mathrm{~nm}$, for the one-dimensional random medium with 5000 solid layers with thickness $d_{s}$ following a uniform distribution between $\left\langle d_{s}\right\rangle \pm \Delta d_{s}=30 \pm 10 \mathrm{~nm}$, and with a porosity $\langle\varepsilon\rangle=0.85$, and $n_{s, \omega_{0}}=1.8$. The index of refraction $n_{s, \omega_{0}}$ is attributed to the yttria host, and the index of extinction $\kappa_{s, \omega_{0}}$ attributed to the ytterbium dopant. The dopant concentration is $4.1 \times 10^{20} \mathrm{~cm}^{-3}$, and the absorption cross section is $0.4 \times 10^{-20} \mathrm{~cm}^{2}$. Thus the spectral absorption coefficient of the crystal is 
TABLE I. Parameters and properties for simulation of luminescence quenching.

\begin{tabular}{lll}
\hline \hline Average porosity & $\langle\varepsilon\rangle$ & 0.85 \\
Incident wavelength & $\lambda_{0}$ & $906 \mathrm{~nm}$ \\
Luminescent wavelength & $\lambda_{l}$ & $996 \mathrm{~nm}$ \\
Solid layer mean thickness & $\left\langle d_{s}\right\rangle$ & $30 \mathrm{~nm}$ \\
Solid layer-thickness spread & $\Delta d_{s}$ & $10 \mathrm{~nm}$ \\
Number of solid layers & $N / 2$ & 5000 \\
Index of refraction for solid & $n_{s}$ & 1.8 \\
Index of extinction for solid & $\kappa_{s}$ & $1.2 \times 10^{-5}$ \\
Ambient temperature & $T_{0}$ & $300 \mathrm{~K}$ \\
Optical-phonon energy & $E_{\mathrm{p}, \text { optical }}$ & $1635 \mathrm{~cm}^{-1}$ \\
Radiative decay rate & $\gamma_{\mathrm{r}}$ & $9551 / \mathrm{s}$ \\
Absorption coefficient for thermal emission & $\bar{\sigma}_{a}$ & $166661 / \mathrm{m}$ \\
\hline \hline
\end{tabular}

$$
\begin{aligned}
\sigma_{a, \omega_{0}, c} & =4.1 \times 10^{20}\left(\mathrm{~cm}^{-3}\right) \times 0.4 \times 10^{-20}\left(\mathrm{~cm}^{2}\right) \\
& =1.64 \mathrm{~cm}^{-1},
\end{aligned}
$$

and $\kappa_{s, \omega_{0}}$ is

$$
\kappa_{s, \omega_{0}}=\frac{\sigma_{a, \omega_{0}, c} \lambda_{0}}{4 \pi}=1.2 \times 10^{-5} .
$$

There are infinite possible realizations for this model composite, and the field results for one of them are shown in Fig. 5. As evident, there is a field enhancement, i.e., there is a peak in the field inside the medium and this peak can be much larger than the incident field, in most realizations. ${ }^{17}$ In periodic porous media, the field is also periodic, resulting in no isolated peaks inside the media (even if the field in this case can also be higher than the incident field). The physical basis of field enhancement is electromagnetic wave interference. In this random multilayer system, the waves will multiply transmit and reflect at all the interfaces, and interfere with each other. At some location for some realization, the interference is so ideally constructive that it results in an extremely large field. Thus this large field enhancement is solely attributed to random porous structure, and cannot be observed in homogeneous or periodic media. Note that the coherence condition (the medium size is smaller than the coherence length) must be satisfied to observe the field enhancement. The coherence length is $\lambda^{2} / \Delta \lambda$ for a central wavelength $\lambda$ and a spectrum width $\Delta \lambda .^{24}$ In this study we use a monochromatic wave, thus satisfying the coherence

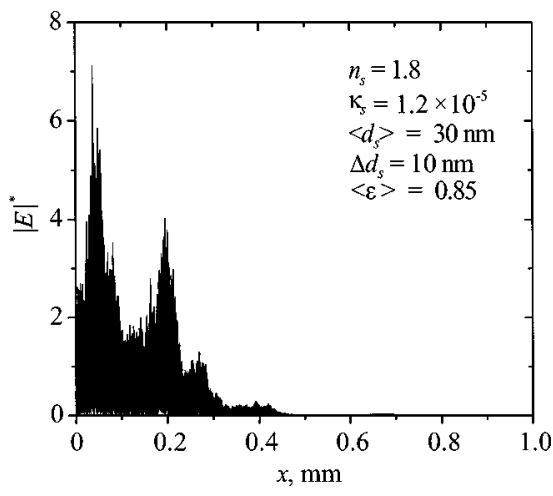

FIG. 5. Typical distribution of dimensionless field in the random, nanoparticle medium with $L=1 \mathrm{~mm}$. $|E|^{*}$ is normalized using the incident field.

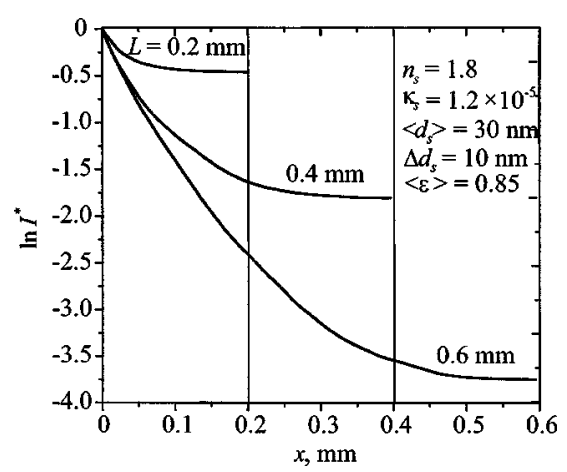

FIG. 6. The decay of the dimensionless intensity with respect to location within the layer system, for thicknesses of $0.2,0.4$, and $0.6 \mathrm{~mm}$, respectively. The dimensionless intensity $I^{*}$ is defined as $I^{*}=I(x) / I(x=0)$.

condition ( $\Delta \lambda$ is zero and coherence length infinite). The coherence length of many lasers is several kilometers, satisfying the coherence condition. More detail on field enhancement and photon localization can be found in Ref. 17.

It is interesting to notice that the penetration depth begins to depend on the sample size $L$ for this random multilayer. The penetration depth is defined as the distance where the intensity decays to $1 / e$ of the initial intensity. To investigate the expectation intensity decay profile of a sample, the intensity profiles of a large number of realizations with the same $L$ are calculated and the ensemble average is made. Shown in Fig. 6 is the expectation decay profile of the dimensionless intensity of samples with the thicknesses of $0.2,0.4$, and $0.6 \mathrm{~mm}$, respectively. It is evident that initially the intensity decays exponentially, and then decays much slower. This is because the near surface region has a stronger coherent interference effect and thus a larger absorption. As a result of the presence of the slow decay region, the penetration depth becomes sample size dependent. A very thin film has a larger penetration depth, because the exponential decay region is too small, and the slow decay region moves towards the surface. As the film thickness increases, the penetration depth decreases and finally approaches a constant value, because the exponential decay region is large enough. The absorption coefficient of the multilayer is the reciprocal of the penetration depth, and is

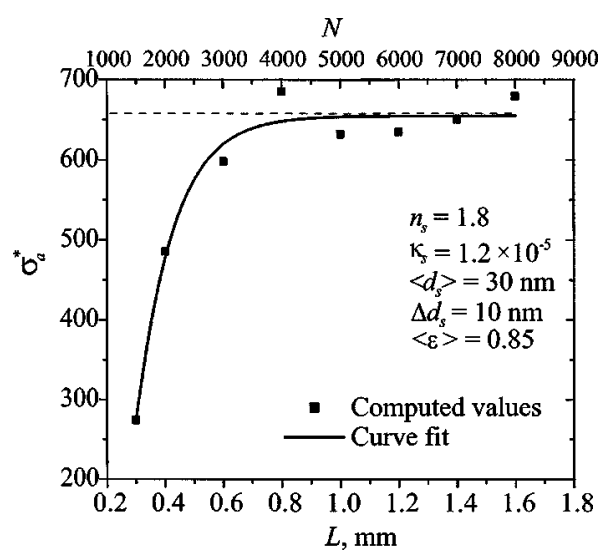

FIG. 7. The variation of the dimensionless absorption coefficient with respect to the sample size. The dimensionless absorption coefficient $\sigma_{a}^{*}$ is defined as $\sigma_{a}^{*}=\sigma_{a} /[4 \pi \kappa / \lambda(1-\langle\varepsilon\rangle)]$. 


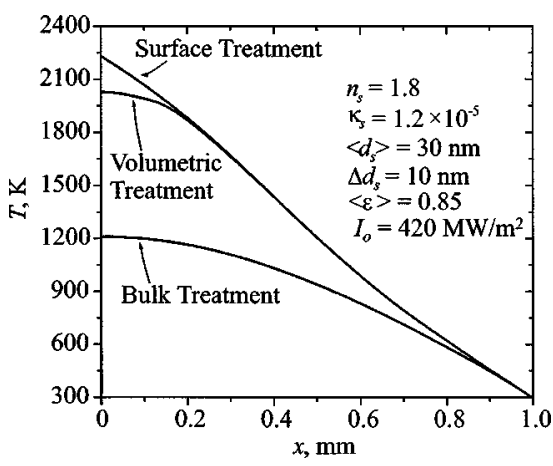

FIG. 8. The predicted temperature distribution using surface, volumetric, and bulk treatments.

normalized against the crystal values to get the dimensionless absorption coefficient. This is shown in Fig. 7. As expected, it is smaller in thin samples, and approaches a constant value as the sample is thick enough.

It should be noted that this rather counterintuitive result is due to the porous medium (parallel layers) used. In homogeneous structures, the intensity follows a rigorous exponential decay along the beam direction, and the penetration depth is well defined. However, in the layered, onedimensional structure considered in this manuscript, the intensity does not follow an ideal exponential decay, as shown in Fig. 6. This is due to the interference. Thus, the penetration depth becomes size dependent.

\section{B. Luminescence quenching and thermal emission}

Using the theoretical model developed above, the steady-state temperature distribution, luminescent, and thermal emission can be calculated given the irradiation intensity. Before this can be done, the temperature dependence of the nonradiative decay rate $\gamma_{\mathrm{nr}}(T)$ needs to be determined. The lifetime measurements ${ }^{14}$ suggest that the nonradiative decay is a six-phonon relaxation process, with the energy of $1635 \mathrm{~cm}^{-1}$ per phonon. However, this is not clear, since the optical phonon of yttria has not yet been fully measured or analyzed [e.g., molecular dynamics (MD)], although part of the phonon spectrum has been reported in Ref. 25. As discussed, since the absorption of incident radiation occurs nonuniformly within the multilayer system, the resulting temperature distribution is different from those predicted using a

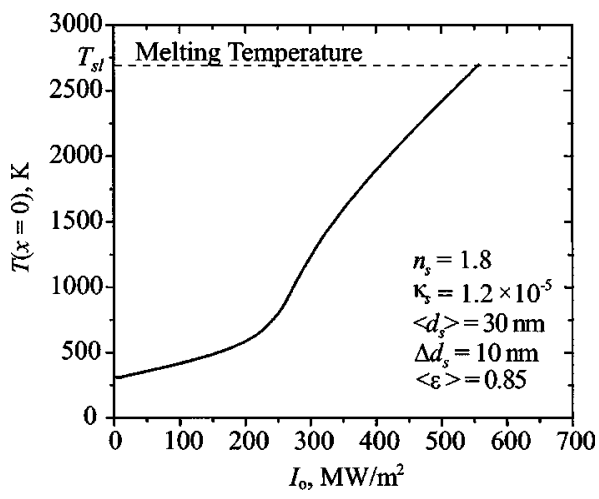

FIG. 9. The variation of the surface temperature with respect to irradiation intensity.

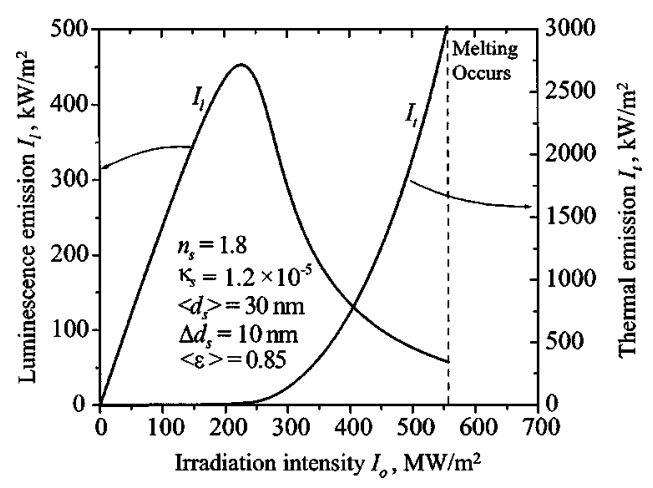

FIG. 10. The variation of the luminescent and thermal emission intensities with respect to irradiation intensity.

surface or a uniform treatment. This is shown in Fig. 8, showing the surface treatment results in a higher surface temperature and the bulk (or uniform) treatment results in a much lower surface temperature.

The variations of the surface temperature, luminescent, and thermal emission intensities, with respect to the irradiation intensities, are shown in Figs. 9 and 10. It is clear that initially the luminescent emission increases linearly with temperature, and then experiences a quenching. This is due to the temperature dependence of the luminescent quantum yield. The initial linear increase in $I_{l}$ is indicated by Eq. (18), because the luminescent quantum yield $\eta$ defined in Eq. (19) is a weak function of $T$ at low temperatures, and $\dot{s}_{0}$ is proportional to the irradiation. In contrast, at high temperatures the decay rate increases rapidly with the temperature, and $\eta$ is also a rapidly decaying function of the temperature. Thus the luminescence emission is quenched. The total thermal emission has the blackbody behavior, and is proportional to $T^{4}$, causing the emission intensity to increase rapidly at high irradiations. It should be noted that melting occurs when the melting temperature is reached. At the phase transition, the surface temperature will take a sudden jump to a higher stable state, because the reflection of the liquid phase is not as large as the solid multilayer. Then the thermal emission is considered bistable.

\section{CONCLUSIONS}

A consistent model is developed to analyze the luminescence quenching of random multilayer systems, by considering the interaction, transition, and transport of basic energy carriers. The coherent wave treatment is used for the photon absorption, and field enhancement and size-dependent penetration depth are predicted. The nonradiative decay is identified as a multiphonon relaxation process, and the luminescent quantum yield is highly temperature dependent, causing the luminescence quenching. Qualitative agreement is found with the luminescent and thermal emissions of irradiated nanopowder. ${ }^{26}$

\section{ACKNOWLEDGMENTS}

The support of Rackham School of Graduate Studies/ Vice President for Research, the University of Michigan, 
through a research grant, is greatly appreciated. Discussions with Professor Stephen Rand have been very helpful and are greatly appreciated.

${ }^{1}$ S. Coffa, G. Franzo, F. Priolo, A. Polman, and R. Serna, Phys. Rev. B 49, 16313 (1994).

${ }^{2}$ J. Palm, F. Gan, B. Zheng, J. Michel, and L. C. Kimerling, Phys. Rev. B 54, 17603 (1996).

${ }^{3}$ W. Ryba-Romanowski, S. Golab, G. Dominiak-Dzik, M. N. Palatnikov, and V. Sidorov, Appl. Phys. Lett. 78, 3610 (2001).

${ }^{4}$ T. Makino, K. Tamura, C. H. Chia, Y. Segawa, M. Kawasaki, A. Ohtomo, and H. Koinuma, J. Appl. Phys. 93, 5929 (2003).

${ }^{5}$ H. M. Gibbs, Optical Bistability: Controlling Light with Light (Academic, Orlando, 1985).

${ }^{6}$ S. T. Feng and E. A. Irene, J. Appl. Phys. 72, 3897 (1992).

${ }^{7}$ M. Hoffmann, P. Kopka, T. Grob, and E. Voges, Electron. Lett. 34, 207 (1998).

${ }^{8}$ A. Boussekou, G. Molnar, P. Demont, and J. Menegotto, J. Mater. Chem. 34, 2069 (2003).

${ }^{9}$ A. Kuditcher, M. P. Hehlen, C. M. Florea, K. W. Winick, and S. C. Rand, Phys. Rev. Lett. 84, 1898 (2000).

${ }^{10}$ M. A. Noginov, M. Vondrova, and B. D. Lucas, Phys. Rev. B 65, 035112 (2001).

${ }^{11}$ A. M. Lawandy, R. M. Balachandran, A. S. L. Gomes, and E. Sauvain, Nature (London) 368, 436 (1994)
${ }^{12}$ H. Cao, Y. G. Zhao, S. T. Ho, E. W. Seelig, Q. H. Wang, and R. P. H. Chang, Phys. Rev. Lett. 82, 2278 (1999).

${ }^{13}$ Y. Feng, J. F. Bisson, J. R. Lu, S. H. Huang, K. Takaichi, A. Shirakawa, M. Musha, and K. Ueda, Appl. Phys. Lett. 84, 1040 (2004).

${ }^{14}$ S. M. Redmond, S. Oliveira, and S. C. Rand, Appl. Phys. Lett. 85, 5517 (2004).

${ }^{15}$ Z. M. Zhang and M. I. Flik, IEEE Trans. Appl. Supercond. 3, 1604 (1993).

${ }^{16} \mathrm{~J}$. A. Kong, Electromagnetic Wave Theory (EMW Publishing, Cambridge, 2000).

${ }^{17}$ X. L. Ruan and M. Kaviany, Microscale Thermophys. Eng. 9, 63 (2005).

${ }^{18}$ M. P. Hehlen, H. U. Gudel, Q. Shu, and S. Rand, J. Chem. Phys. 104, 1232 (1995).

${ }^{19}$ T. Miyakawa and D. L. Dexter, Phys. Rev. B 1, 2961 (1970).

${ }^{20}$ J. Costa, P. Roura, J. R. Morante, and E. Bertran, J. Appl. Phys. 83, 7879 (1998).

${ }^{21}$ R. Siegel and J. R. Howell, Thermal Radiation Heat Transfer, 4th ed. (Taylor \& Francis, New York, 2002).

${ }^{22}$ Y. S. Touloukian and C. Y. Ho, in Thermophysical Properties of Matter, The TPRC Data Series (IFI-Plenum, New York, 1970).

${ }^{23}$ M. Kaviany, Principles of Heat Transfer (Wiley, New York, 2002).

${ }^{24}$ E. Hecht, Optics (Addison-Wesley, San Francisco, CA, 2002).

${ }^{25}$ G. Schaack and J. A. Koningstein, J. Opt. Soc. Am. 60, 1110 (1970).

${ }^{26}$ S. Redmond, S. C. Rand, X. L. Ruan, and M. Kaviany, J. Appl. Phys. 95, 4069 (2004). 